Reprod. Nutr. Dévelop., 1985, 25 (4 A), 717-727.

\title{
Absence d'effet de la vitamine $D$ sur la phytase et la phosphatase alcaline intestinales : relation avec l'absorption du phosphore phytique chez le porc
}

\author{
Nicole FONTAINE $\left({ }^{*}\right)$, Annie FOURDIN $\left({ }^{* *}\right)$, A. POINTILLART \\ avec la collaboration technique de B. CAYRON, Pierrette CAMUS, Colette COLIN
}

$\left.{ }^{*}\right)$ C.N.R.S., $\left(^{* *}\right)$ Stagiaire de $3^{e}$ cycle, Station de Recherches de Nutrition, I.N.R.A. 78350 Jouy en Josas, France.

Summary. Effects of vitamin $D$ on intestinal phytase and alkaline phosphatase and its relationship with phytate-phosphorus absorption in pigs.

At least two-thirds of the phosphorus ingested by pigs is in the form of phytates. Two intestinal vitamin $D$-sensitive enzymes, alkaline phosphatase and phytase, might be involved in phytate-P digestion. The effects of dietary vitamin $D$ upon the two intestinal phosphatases and $P$ utilization in pigs fed a high phytate- $P$ diet are reported here. Fourteen vit. D-depleted pigs (25-hydroxy vit. D : about $2 \mathrm{ng} / \mathrm{ml}$ plasma) were divided into two groups fed the same basal diet containing $0.6 \% \mathrm{P}$ (of which $80 \%$ was phytic) and $0.6 \%$ $\mathrm{Ca}$. One group (+ D) was supplemented for 5 weeks with vit. D (1 $000 \mathrm{IU} \mathrm{D}_{3} / \mathrm{kg}$ diet $)$ and the other $(-D)$ received none.

$P$ absorption and retention was two times higher in $+D$ pigs than in $-D$ animals (balance technique), and tibia $X$-ray pictures showed a lower bone density with the - D diet than with the $+D$ diet. Surprisingly, vit. D supplementation had no effect on either of the mucosal enzymes (phytase and alkaline phosphatase). It may be concluded that vit. D improves phytate-P absorption via a mechanism which does not involve an increase in the activity of the intestinal phosphatases.

\section{Introduction.}

Chez le porc, l'apport alimentaire de phosphore est, au moins pour les deux tiers, sous forme de phytates provenant essentiellement des céréales et des tourteaux (Bagheri et al., 1982). Au niveau intestinal, deux phosphatases, la phytase (E.C.3.1.3.8.) et la phosphatase alcaline (E.C.3.1.3.1.) sont susceptibles d'intervenir dans I'hydrolyse des phytates (McCuaig et Motzok, 1972 ; Davies, Ritcey et Motzok, 1970 ; Davies et Flett, 1978) et dans l'absorption du phosphore (Shirazi, Beechey et Butterworth, 1981 ; Bachelet, Lacour et Ulmann, 1982 ; Kempson et al., 1979 ; Birge et Avioli, 1981). Chez le rat ou le poulet, l'activité de l'une et I'autre enzymes augmente avec l'apport de vitamine D tant in vivo (Steenbock et al., 1953 ; Pileggi, De Luca et Steenbock, 1955 ; Davies, Ritcey et Motzok, 1970 ; Roberts et Yudkins, 1961 ; Bhandari, 1980 ; Valinietse et Bauman, 1984) qu'in 
vitro (Peterlik et Wasserman, 1980 ; Chan et Atkins, 1983 ; Haussler, Nagode et Rasmussen, 1970 ; Bikle et al., 1979 ; Bachelet, Ulmann et Lacour, 1979). Certains auteurs (Bachelet, Lacour et Ulmann, 1982 ; Birge et Avioli, 1981) attribuent un rôle à la phosphatase alcaline dans le transport du phosphore à travers la muqueuse intestinale du fait d'une part de l'existence de substances inhibitrices communes (Haussler, Nagode et Rasmussen, 1970 ; Bachelet, Lacour et Ulmann, 1982) et, d'autre part de leur commune stimulation par le métabolite le plus actif de la vitamine $D$, le 1,25-dihydroxycholécalciférol $\left(1,25(\mathrm{OH})_{2} \mathrm{D}_{3}\right)$ (Peterlik et Wasserman, 1980 ; Matsumoto, Fontaine et Rasmussen, 1980).

L'augmentation des concentrations en $1,25(\mathrm{OH})_{2} \mathrm{D}$ lors de carence en phosphore est un phénomène connu chez le rat et chez le poulet (De Luca, Tanaka et Baxter, 1975) qui a été récemment confirmé, chez le porc, par l'observation d'une corrélation négative étroite entre la concentration plasmatique de $1,25(\mathrm{OH})_{2} \mathrm{D}_{3}$ et la phosphatémie (Pointillart, Fontaine et Thomasset, 1984a), lorsque les régimes sont pauvres en phosphore. Par contre, certains résultats obtenus aussi chez le porc, bien qu'ils suggèrent une réponse de la vitamine $D$ à la carence en phosphore (augmentation de la phosphatase alcaline et de la CaBP duodénales, élévation de la concentration plasmatique de 25 hydroxy-vitamine D), ne permettent pas d'impliquer les phosphatases (phytase et phosphatase alcaline) dans l'augmentation de l'absorption du phosphore mesurée in vivo par la méthode des bilans (Pointillart, Fontaine et Thomasset, 1984b).

En conséquence, l'influence de la vitamine $D$ sur la phytase et la phosphatase alcaline intestinales en relation avec l'absorption du phosphore phytique a été étudiée chez des porcs préalablement carencés en vitamine $D$.

\section{Matériel et méthodes.}

1. Animaux et régimes. - Quatorze porcelets Large-White, pris au sevrage, pesant $7 \pm 0,2 \mathrm{~kg}$, âgés de 26 jours, ont été placés à l'obscurité et ont reçu pendant une période d'environ 2 mois, un aliment pré-expérimental. Celui-ci était identique à l'aliment classique utilisé lors du sevrage, riche en calcium $(1,7 \%)$, mais non supplémenté en vitamine $D$ (tabl. 1). Progressivement (sur une période de deux semaines), l'aliment expérimental carencé en vitamine $D$ (tabl. 1) et dépourvu de phosphore minéral ( $\mathrm{P}$ total : $0,6 \%$ dont $80 \%$ sous forme phytique) a été substitué à l'aliment sevrage. Finalement, les porcs ont été répartis en 2 lots de 7, I'un ayant continué à recevoir l'aliment expérimental carencé $(-D)$ et l'autre, ce même aliment supplémenté avec 1000 ui de vit. $D_{3}$ par $\mathrm{kg}(+\mathrm{D})$ ). Sur 4 animaux par lot, alimentés de façon appariée, un bilan de 10 jours a été réalisé dans les deux semaines précédant le sacrifice, effectué un mois après le début de l'essai.

L'aliment expérimental contenait $0,6 \%$ de $P$, teneur voisine des recommandations usuelles (Gueguen et Perez, 1981) dans cette espèce; il ne présentait aucune activité phytasique, laquelle aurait pu interférer dans l'utilisation digestive du phosphore, comme il a été récemment montré (Pointillart, Fontaine et Thomasset, 1984b). L'apport de calcium a été réduit $10,6 \%$ contre $0,95 \%$ recom- 
mandé) pour prendre en compte la relative indisponibilité du $P$ phytique, susceptible d'entraîner une mauvaise rétention calcique (Pointillart, Fontaine et Thomasset, $1984 a$ et $b$ ).

TABLEAU 1

Composition des aliments $(\%)$.

\begin{tabular}{lclc}
\hline \multicolumn{2}{c}{ Aliment pré-expérimental } & \multicolumn{2}{c}{ Aliment expérimental } \\
\hline Orge & 18 & Avoine décortiquée & 56,4 \\
Blé & 18 & Tourteau de soja 50 & 20 \\
Maïs & 18 & Tourteau de colza & 20 \\
Tourteau de soja 50 & 23 & Mélasse & 2 \\
Lait écrémé en poudre & 15 & Carbonate de calcium & 1 \\
Suif 1er jus & 3 & Chlorure de sodium & 0,4 \\
Carbonate de calcium & 2,4 & CMVb & 0,15 \\
Phosphate bicalcique & 1,3 & & \\
Chlorure de sodium & 0,3 & & 0,59 \\
CMVa & 1 & & 0,58 \\
\hline Calcium & 1,68 & & 0,46 \\
P total (A) & 0,73 & & 0,80 \\
P phytique (B) & 0,40 & & \\
B/A & 0,55 & & \\
\hline
\end{tabular}

CMVa (CMVb), apports en mg/kg d'aliment : $\mathrm{Cu}: 10$ (125), $\mathrm{Zn}: 85$ (150). Fe : 60 (80). Mn : 24 (30), Co : 2 (1), I: 0,6 (0,6) ; vitamines par kg d'aliment : A : 5000 ui (ib.), E : $20 \mathrm{mg}$ (ib.), B1: $2 \mathrm{mg}$ (ib.), B2 : $10 \mathrm{mg}$ (5), B6:10 mg (ib.), C : $20 \mathrm{mg}$ (ib.), B12:50 $\mathrm{gg}$ (ib.), pantothénate de Ca : $40 \mathrm{mg}$ (20), chlorhydrate de choline : $1000 \mathrm{mg}(750)$, niacine : $40 \mathrm{mg}(20)$. Vitamine $D_{3}$ : pas de supplémentation sauf régime $+\mathrm{D}(\mathrm{CMVb}): 1000 \mathrm{ui} / \mathrm{kg}$ rajoutées à l'aliment expérimental ( - D).

L'état de carence en vitamine $D$ a été vérifié par le dosage du métabolite circulant servant généralement de témoin (Garabedian, 1984), le 25(OH)D (méthode de Preece et al., 1974). Au sevrage, la concentration plasmatique était de $4 \mathrm{ng} / \mathrm{ml}$ $(4,1 \pm 1,2)$; pour la majorité des porcelets $(9 / 14)$, elle diminuait pour atteindre environ $2 \mathrm{ng} / \mathrm{ml}(1,7 \pm 0,1)$ la semaine ayant précédé la mise en lot.

2. Prélèvements et mesures. - A l'abattage, des échantillons de muqueuse intestinale ont été prélevés à partir du duodénum $(20 \mathrm{~cm})$, du jéjunum $(40 \mathrm{~cm}$, partie médiane), de l'iléon (segment distal, $40 \mathrm{~cm}$ ) du cæcum (proximal : $40 \mathrm{~cm}$ ) et du côlon (fragment distal, $40 \mathrm{~cm}$ ) selon un protocole précédemment décrit (Pointillart, Fontaine et Thomasset, 1984b). L'activité de la phosphatase alcaline est mesurée à $\mathrm{pH} 9,8$, à $37^{\circ} \mathrm{C}$ à l'aide de paranitrophényl phosphate (Bessey, Lowry et Brock, 1946) et celle de la phytase à pH 8 , à $37^{\circ} \mathrm{C}$, avec le substrat phytate de sodium (Bitar et Reinhold, 1972). Les résultats ont été exprimés en milli unités internationales par $\mathrm{mg}$ de protéines de la muqueuse, estimées par la méthode de Lowry et a/. (1951) (1 ui $=1 \mu$ Mole de $\mathrm{P}$ libérée par min à $37^{\circ} \mathrm{C}$ ).

Tibias et péronés ont été utilisés pour déterminer la composition minérale des os (cendres, $\mathrm{Ca}, \mathrm{P}$ ) et l'opacité radiographique. En outre, la carence en vitamine $D$ pouvant interférer dans le métabolisme lipidique (Anonyme, 1984), les échantillons de métaphyses tibiales (distales et proximales) ont été préalablement dégraissés avant leur minéralisation. La résorption osseuse a été estimée par 
I'intermédiaire du dosage de l'hydroxyproline dans les urines récoltées au cours du bilan (Szymanowicz, Randoux et Borel, 1979). Les phytates du régime ont été dosés par la méthode d'Oberleas (1971) et son activité phytasique par celle de Bitar et Reinhold (1972) à pH 5. Le sang collecté à l'abattage a permis la mesure des concentrations plasmatiques de calcium, de phosphore inorganique, de 25(OH)D et de l'activité de la phosphatase alcaline (phosphatasémie, exprimée en unités Bessey-Lowry/l). Le test $t$ de Student a permis la comparaison des moyennes (présentées suivies de leurs écart-types).

\section{Résultats.}

1. Absorption et rétention du phosphore (tabl. 2). - L'absorption et la rétention du phosphore étaient significativement plus élevées $(\times 2$ environ) dans le groupe ayant reçu un apport vitaminique $D$ que dans celui qui en a été privé jusqu'à la fin de l'expérience, qu'elles soient exprimées en valeurs absolues $(\mathrm{g} / \mathrm{j})$ ou relatives (en \% de l'ingéré). La phosphaturie était comparable dans les 2 groupes, elle est restée, pour l'ensemble des animaux, excessivement faible, puisqu'elle était inférieure à $100 \mathrm{mg} /$ jour, soit moins de 1,5\% de l'ingéré. A noter également qu'avec le régime $+D$, l'absorbé (en $\mathrm{g} / \mathrm{j}$ ) correspondait à deux fois la quantité de phosphore non phytique ingérée. Une proportion importante de $P$ phytique a donc été absorbée, ce qui n'était pas le cas avec le régime carencé.

TABLEAU 2

Bilan du phosphore

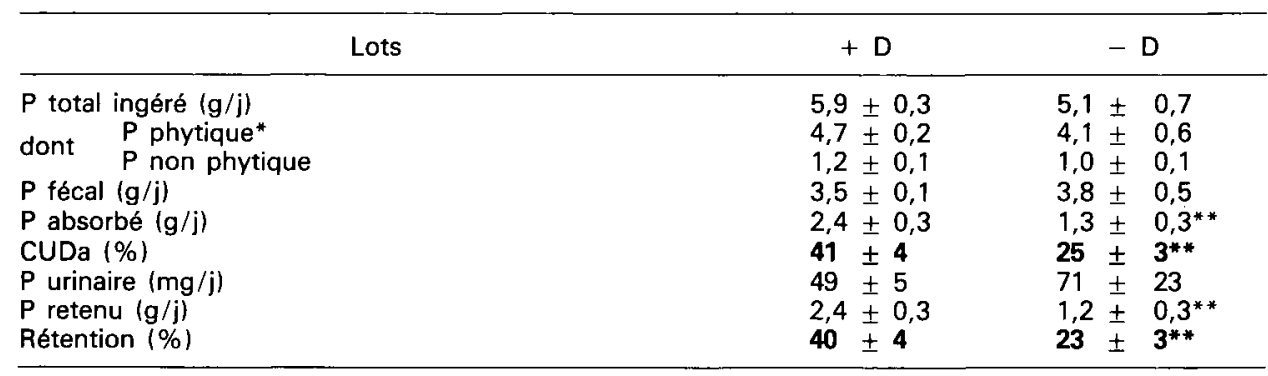

* Calculé à partir du dosage de $P$ phytique de l'aliment. P non phytique : phosphore organique apporté par les matières premières (avoine, soja, colza), l'aliment n'étant pas supplémenté en $P$ minéral (cf. texte).

** $\mathbf{P}<0,01$. CUDa : coefficient d'utilisation digestive apparent (absorbé \% ingéré).

2. Activités phosphatasiques intestinales (tabl. 3). - Quels que soient les segments considérés, la supplémentation en vitamine D n'a eu aucun effet sur l'activité des deux enzymes, phytase ou phosphatase alcaline. L'activité phytasique était extrêmement faible comparée à celle de la phosphatase alcaline : en moyenne moins de $1 \mathrm{~m}$ ui (par $\mathrm{mg}$ de protéines de la muqueuse), soit, dans l'intestin grêle, 100 fois moins que pour l'autre phosphatase. 
Le jéjunum était la partie intestinale où se manifestait la plus forte activité phosphatasique alcaline, duodénum et iléon ayant eu des activités comparables et 2 ou 3 fois plus faibles que dans le jéjunum. Celles du gros intestin étaient très faibles comparativement à celles du grêle (10 fois moins). II n'y avait pas de corrélation significative entre les activités phosphatasiques intestinales et les quantités de phosphore absorbées ( $r=0,22$ au niveau jéjunal, NS).

\section{TABLEAU 3}

Activités phosphatasiques de la muqueuse intestinale (en $\mathrm{m} \mathrm{ui/mg} \mathrm{de} \mathrm{protéines).}$

\begin{tabular}{lrrrrr}
\hline & Duodénum & Jéjunum & Iléon & Caecum & Côlon \\
\hline $\begin{array}{l}\text { Phosphatase alcaline (E.C.3.1.3.1.) } \\
\text { + D }\end{array}$ & $91 \pm 24$ & $250 \pm 40$ & $130 \pm 17$ & $10 \pm 1$ & $12 \pm 1$ \\
- D & $74 \pm 15$ & $238 \pm 33$ & $96 \pm 19$ & $10 \pm 1$ & $10 \pm 1$ \\
Phytase (E.C.3.1.3.8.) & & & & & \\
+ D & $0,8 \pm 0,1$ & $1,2 \pm 0,2$ & $0,6 \pm 0,1$ & $0,6 \pm 0,1$ & $1,1 \pm 0,2$ \\
- D & $0,7 \pm 0,1$ & $0,9 \pm 0,3$ & $0,4 \pm 0,1$ & $0,7 \pm 0,1$ & $0,8 \pm 0,3$ \\
\hline
\end{tabular}

Aucun écart significatif entre régimes. Pour E.C.3.1.3.1. les valeurs jéjunales sont supérieures aux autres $(P<0,01)$, celles du gros intestin (caecum ou côlon) sont inférieures à toutes celles du grêle $(P<0,01)$.

3. Phosphatasémie, minéraux et 25/OHID plasmatiques (tabl. 4). - Les animaux carencés se caractérisaient par une hypocalcémie très importante (chute de $30 \%$ de la valeur témoin), une hypophosphatémie (de $30 \%$ également), une hyperphosphatasémie considérable (valeur témoin $\times 3$ ) et une concentration en $25(\mathrm{OH}) \mathrm{D}$ circulante de $0,21 \mathrm{ng} / \mathrm{ml}$, significativement diminuée $(\mathrm{P}<0,01)$ par rapport à la valeur pré-expérimentale $(1,7 \mathrm{ng} / \mathrm{ml}$ ) (plusieurs valeurs étaient à la limite de sensibilité du dosage ou indétectables). Au contraire, chez les porcs supplémentés, le 25(OH)D était considérablement augmenté (valeur initiale $\times 10$ ).

4. Aspects osseux. - Les tibias du lot + D étaient significativement $(\mathrm{P}<0,05)$ plus longs $(10,2 \pm 0,2 \mathrm{~cm})$ et plus lourds $(39 \pm 2 \mathrm{~g})$ que ceux du lot

\section{TABLEAU 4}

Concentrations plasmatiques de calcium, phosphore inorganique, 25/OH)D et phosphatasémie.

\begin{tabular}{lcc}
\hline \multicolumn{1}{c}{ Lots } & $+\mathrm{D}$ & $-\mathrm{D}$ \\
\hline Phosphore inorganique $(\mathrm{mg} / 100 \mathrm{ml})$ & $7,5 \pm 0,7$ & $5,1 \pm 0,2^{* *}$ \\
Calcium $(\mathrm{mg} / 100 \mathrm{ml})$ & $11,59 \pm 0,31$ & $7,99 \pm 0,14^{* *}$ \\
Phosphatase alcaline* (unités B.L./I) & $2,9 \pm 0,2$ & $9,1 \pm 1,0^{* *}$ \\
$25(\mathrm{OH}) \mathrm{D}(\mathrm{ng} / \mathrm{ml})$ & $16,9 \pm 4,1$ & $0,21 \pm 0,06^{* *}$ \\
\hline
\end{tabular}

* 1 u B.L. $=1 \mathrm{mmole}$ de p. nitrophénol $/ \mathrm{h} / \mathrm{l}$ à $38^{\circ} \mathrm{C}$.

** $P<0,01$. 
- $D(9,5 \pm 0,2 \mathrm{~cm}$ et $33 \pm 2 \mathrm{~g}$, respectivement $)$ ce qui allait de pair avec une vitesse de croissance supérieure du lot ayant reçu de la vitamine $D(+D: 590 \pm$ $60 \mathrm{~g} / \mathrm{j},-\mathrm{D}: 411 \pm 49, \mathrm{P}<0,05)$.

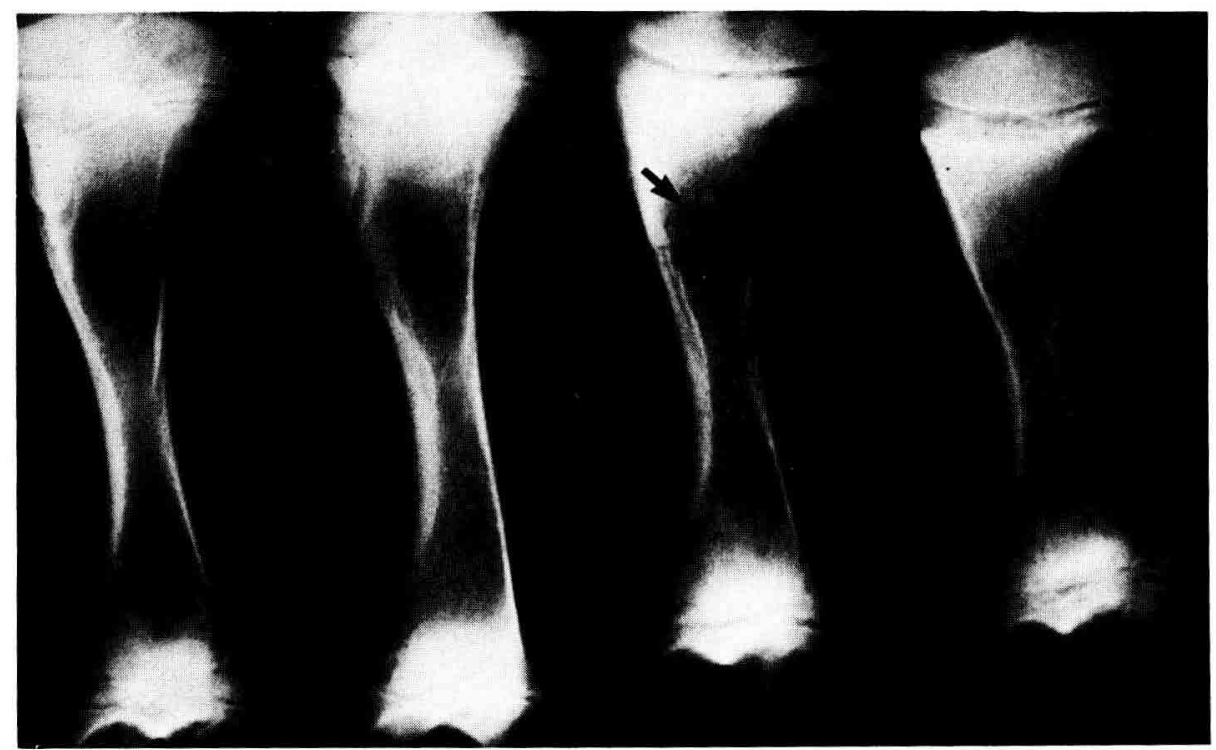

FIG. 1. - Radiographies des tibias.

A gauche : deux os du lot $+\mathrm{D}$; à droite : 2 os du lot $-\mathrm{D}$, la flèche indique une zone de fracture.

La comparaison des clichés radiographiques (fig. 1) met clairement en évidence une opacité supérieure des tibias du lot $+D$, ainsi que des fractures $(2$ porcs sur 7$)$ pour les os du lot $-D$ seulement. Ces résultats ne sont que partiellement confirmés par les analyses minérales (tabl. 5). Rapportées à l'os sec non

TABLEAU 5

Composition minérale des os.

\begin{tabular}{|c|c|c|c|}
\hline & Cendres & Calcium & Phosphore \\
\hline Póroné entier $\left\{\begin{array}{l}+D \\
-D\end{array}\right.$ & $\begin{array}{l}49 \pm 1 \\
49 \pm 1\end{array}$ & $\begin{array}{l}\text { de la matière } \\
19 \pm 0,4 \\
19 \pm 0,7\end{array}$ & $\begin{array}{l}8,4 \pm 0,7 \\
7,6 \pm 0,2\end{array}$ \\
\hline $\begin{array}{l}\text { Tibia } \\
\text { épiphyse } \quad\left\{\begin{array}{l}+D \\
-D\end{array}\right.\end{array}$ & $\begin{array}{l}36 \pm 1 \\
35 \pm 1\end{array}$ & $\begin{array}{l}13 \pm 0,5 \\
13 \pm 0,4\end{array}$ & $\begin{array}{l}5,1 \pm 0,2 \\
5,1 \pm 0,2\end{array}$ \\
\hline$\left\{\begin{array}{l}+D \\
-D\end{array}\right.$ & $\begin{array}{l}63 \pm 1 \\
63 \pm 1\end{array}$ & $\begin{array}{l}21 \pm 0,3 \\
21 \pm 0,9\end{array}$ & $\begin{array}{l}7,9 \pm 0,2 \\
7,9 \pm 0,2\end{array}$ \\
\hline & \multicolumn{3}{|c|}{ (en \% de la MS dégraissée) } \\
\hline métaphyse $\left\{\begin{array}{l}+D \\
-D\end{array}\right.$ & $\begin{array}{l}39 \pm 2 \\
39 \pm 4\end{array}$ & $\begin{array}{l}18 \pm 0,3 \\
14 \pm 0,7^{* *}\end{array}$ & $\begin{array}{l}6,2 \pm 0,3 \\
6,0 \pm 0,2\end{array}$ \\
\hline
\end{tabular}

** $P<0,01$. 
dégraissé, les teneurs en cendres, en phosphore et en calcium du péroné, des diaphyses et épiphyses tibiales n'étaient pas différentes d'un lot à l'autre. Par contre, le contenu total en calcium (mais pas celui de phosphore), calculé sur le péroné entier, était plus élevé chez les supplémentés $(+D: 600 \pm 40 \mathrm{mg},-D$ : $500 \pm 30 \mathrm{mg}$ de $\mathrm{Ca}, \mathrm{P}<0,05)$, ce qui est à relier à une teneur accrue en matière sèche $(+D: 58 \pm 2 \%,-D: 53 \pm 1 \%$ de l'os frais, $P<0,01)$. Rapportée à l'os sec dégraissé, la teneur en calcium des métaphyses tibiales était significativement plus faible, chez les carencés, mais non la teneur en phosphore (tabl. 5). Un écart significatif existait également au niveau des teneurs en calcium rapportées aux cendres, mais pour les métaphyses tibiales seulement $1+D: 38,7 \pm 0,9 \%$, - $D: 35,7 \pm 0,8 \%, C a \%$ cendres, $P<0,01)$. Enfin, l'ostéolyse était accrue chez les carencés comme la comparaison des hydroxyprolinuries l'indique : $-D=354 \pm 22 \mathrm{mg} / \mathrm{j}$ et $+D=211 \pm 34, P<0,05$.

\section{Discussion.}

Dans les conditions expérimentales utilisées, c'est-à-dire avec des animaux préalablement carencés, l'introduction dans le régime de vitamine $D$ améliore la minéralisation osseuse et évite les fractures mais surtout l'absorption du phosphore, pour l'essentiel phytique, double sans que les activités des deux phosphatases intestinales, quel que soit le segment considéré, soient augmentées.

L'ensemble des travaux de Moore et Veum $(1982,1983 a, b)$ et de Moore, Reeves et Veum (1984) démontre que, chez le rat, les changements dans l'absorption du phosphore phytique, quand ils existent, ne sont liés ni à la phytase, ni à la phosphatase alcaline de la muqueuse intestinale. Par contre, chez le poulet soumis à un régime carencé en phosphore, l'activité de la phosphatase alcaline dans la muqueuse augmente de $50 \%$, comparée aux témoins (McCuaig et Motzok, 1972). Cela n'est pas le cas chez le rat selon Moore et Veum, alors que Kempson et al. (1979) montrent que la carence en phosphore stimule la phosphatase alcaline de l'intestin $(\times 1,5)$. Enfin, chez le porc recevant un régime pauvre en $P$, la phytase intestinale est inchangée (Moser et al., 1982 ; Pointillart, Fontaine et Thomasset, 1984b) et qui plus est, la phosphatase alcaline duodénale peut doubler sans que cela corresponde à une meilleure absorption du P phytique (Pointillart, Fontaine et Thomasset, 1984b).

L'implication de la phosphatase alcaline dans le transfert de phosphore, au travers de la muqueuse intestinale, est le sujet de nombreuses controverses. Certains (Peterlik et Wasserman, 1980 ; Matsumoto, Fontaine et Rasmussen, 1980) constatent qu'in vitro, chez le poulet, la stimulation du transport de $\mathrm{P}$ par la vitamine $D$ précède celle de la phosphatase alcaline, alors que Bachelet, Lacour et Ulmann (1982) concluent d'un essai sur anse intestinale de rat isolée in situ, que le $1,25(\mathrm{OH})_{2} \mathrm{D}_{3}$ stimulerait le transport de $\mathrm{P}$ par l'intermédiaire d'une activation de la phosphatase alcaline, leur hypothèse se fondant sur une inhibition commune par certaines substances (phénylalanine, lévamisole). Au contraire, Shirazi, Beechey et Butterworth (1981), en utilisant des inhibiteurs de la phosphatase alcaline différents (thio ou imidonucléotides) démontrent que le transfert actif de $\mathrm{P}$ (Nadépendant), dans les vésicules de bordure en brosse, n'est pas modifié. 
En fait, la seule équipe (Moore et Veum) qui a étudié simultanément et l'absorption in vivo du phosphore (notamment phytique) et les activités des deux phosphatases, la phytase et la phosphatase alcaline duodénales, conclut à une absence de relation entre ces enzymes et les quantités absorbées de phosphore total ou phytique. Ces résultats, les seuls obtenus chez le rat in vivo, s'accordent assez bien avec ceux du présent travail et d'autres obtenus précédemment, également chez le porc, et qui montraient des variations dans l'absorption du P phytique indépendantes des deux activités phosphatasiques intestinales (Pointillart, Fontaine et Thomasset, $1984 a$ et $b$ ).

Par contre, l'absence d'effet de la supplémentation en vitamine D sur l'activité des deux phosphatases, chez le porc, est en contradiction avec l'ensemble des résultats antérieurs, obtenus soit chez le poulet, soit chez le rat. La carence en vitamine $D$, en affectant l'intégrité de la muqueuse (McCarthy, Barham et Kumar, 1984), pourrait en diminuer la teneur en protéines, masquant ainsi un éventuel écart des activités enzymatiques qui sont rapportées à cette teneur. Cependant, il ne semble pas qu'il en soit ainsi, puisqu'au niveau duodénal, seul segment où la teneur en protéines différait significativement $(+D: 100 \pm 4$, - $D: 85 \pm 4 \mathrm{mg}$ de protéines/g de muqueuse, $P<0,02)$, l'activité phosphatasique alcaline rapportée à la masse de muqueuse est restée comparable $(+D$ : $6,8 \pm 1,3 ;-D: 6,1 \pm 1,1 \mathrm{ui} / \mathrm{g}$ de muqueuse, $P>0,101$, ainsi que celle de la phytase $(+D: 77 \pm 12,-D: 56 \pm 9 \mathrm{~m}$ ui/g de muqueuse, $P>0,10)$. II pourrait donc s'agir d'une particularité de l'espèce porcine. Toutefois, il a déjà été montré, chez le rat (Krawitt et Korson, 1972) un effet significatif de l'apport de vitamine $D$ sur la phosphatase alcaline duodénale $(\times 3$, comparée aux carencés) qui ne se manifestait pas aux niveaux jéjunal et iléal. Par ailleurs, lors d'un précédent essai (Pointillart, Jay et Fontaine, 1985), il a été observé, chez des porcs devenus rachitiques $(25(\mathrm{OH}) \mathrm{D}<1 \mathrm{ng} / \mathrm{ml})$, des activités phosphatasiques supérieures (phosphatase alcaline jéjunale et iléale : de 200 à $500 \mathrm{~m}$ ui contre 100 à $250 \mathrm{~m}$ ui, tabl. 2) ou égales (phosphatase alcaline duodénale et phytase, quel que soit le segment) à celles décrites, ici, chez les porcs supplémentés en vitamine $D$. Enfin, il a été vérifié, in vitro, sur des prélèvements de muqueuse jéjunale et duodénale provenant de porcs non supplémentés en vitamine $D$, que ni le $1,25(\mathrm{OH})_{2} \mathrm{D}_{3}$ (aux doses de 34 et $85 \mathrm{pg} / \mathrm{ml}$ d'homogénat), ni le 25(OH) $\mathrm{D}_{3}$ (aux doses de 10 et $40 \mathrm{ng} / \mathrm{ml}$ d'homogénat) n'activaient la phytase.

L'hypocalcémie, I'hypophosphatémie, l'hyperphosphatasémie et la disparition presque complète du métabolite témoin de l'état de réplétion vitaminique $D$, le 25(OH)D sont des signes classiques de la carence en vitamine $D$ (Boudailliez, 1984). Il en est de même pour les fractures et l'opacité radiographique diminuée associées à l'hyperrésorption osseuse dont témoigne l'hyperhydroxyprolinurie. La parathormone circulante n'a pas été mesurée, mais, chez les carencés, le poids des parathyroïdes $(90 \pm 8 \mathrm{mg})$ était significativement supérieur $(P<0,05)$ à celui des supplémentés $(65 \pm 7 \mathrm{mg}$ ), ce qui pourrait suggérer une réaction parathyroïdienne ayant stimulé la résorption. Les péronés présentaient une composition normale en minéraux, mais un contenu total de l'os en calcium nettement diminué chez les carencés, conséquence probable de la chute générale de croissance ayant atteint les os comme on l'a vu avec les tibias. Néanmoins, la chute de la 
teneur en calcium des métaphyses tibiales (rapportée à la matière sèche de l'os dégraissé) témoigne également d'un trouble de la minéralisation. II est vraisemblable que la déminéralisation (ou la minéralisation plus faible) apparue chez les porcs - D provienne à la fois de la résorption accrue et d'une formation osseuse inhibée, comme le laissent supposer l'hyperphosphatasémie considérable et les chutes exceptionnelles des quantités de phosphore, mais aussi de celles de calcium retenues ( $-D: 0,5 \pm 0,3 \mathrm{~g} /$ jour, $+\mathrm{D}: 3,1 \pm 0,5 \mathrm{~g}, \mathrm{P}<0,01$ ).

En conclusion, la supplémentation vitaminique $D$ stimule très nettement l'absorption du phosphore et sa rétention même quand sa forme alimentaire est, à $80 \%$, phytique. Cet effet est indépendant de la phytase (E.C.3.1.3.8.) et de la phosphatase alcaline (E.C.3.1.3.1.) de la muqueuse intestinale qui, chez le porc, contrairement au poulet et au rat, apparaissent peu sensibles à l'apport alimentaire de vitamine $\mathrm{D}$.

Reçu en mars 1985. Accepté en mai 1985.

Remerciements. - Les auteurs remercient la Station I.N.R.A. de Rennes St-Gilles pour avoir mis à leur disposition des porcelets au moment du sevrage et pour la formule alimentaire pré-expérimentale ( $M$. Sève), la Fabrique d'Aliments expérimentaux de la Minière (I.N.R.A.) pour la fabrication des régimes, ainsi que Melle Agnès Bourdeau (C.H.U. Necker) pour les radiographies osseuses. Nous remercions également Monique Thomasset et $\mathrm{L}$. Guéguen pour les conseils apportés au cours de ce travail.

\section{References}

ANONYME, 1984. Altered membrane lipid composition : a possible molecular basis of vitamin D action. Nutr. Rev., 42, 357-358.

BACHELET M., LACOUR B., ULMANN A., 1982. Early effects of $1 \alpha, 25$-dihydroxy vitamin $D_{3}$ on phosphate absorption. A role for alkaline phosphatase ? Miner. Electrolyte Metab., 8, 261 266.

BACHELET M., ULMANN A., LACOUR B., 1979. Early stimulation of alkaline phosphatase activity in response to $1 \alpha, 25$-dihydroxycholecalciferol. Biochem. Biophys. Res. Com., 89, 694-700.

BAGHERI S., FONTAINE N., POINTILLART A., GUEGUEN L., 1982. Influence des fibres et des phytates sur l'utilisation des minéraux chez le porc. In Physiologie digestive chez le Porc. Les Colloques de I'I.N.R.A., $n^{0} 12$, Ed. I.N.R.A.

BESSEY O., LOWRY O., BROCK M. J., 1946. A method for the rapid determination of alkaline phosphatase with five cubic millimeters of serum. J. biol. Chem., 164, 321-329.

BHANDARI S. D., 1980. Effect of phytate feeding with and without protein and vitamin D deficiencies on intestinal phytase activity in rat. Indian J. Biochem. Biophys., 17, 309-312.

BIKLE D. D., MORRISSEY R. L., ZOLOCK D. T., HERMAN R. H., 1979. Stimulation of chick gut alkaline phosphatase activity by actinomycin $D$ and 1,25-dihydroxy vitamin $D_{3}$ : evidence for independent mechanisms. J. Lab. clin. med., 94, 88-94.

BIRGE S. J., AVIOLI R. C., 1981. Intestinal phosphate transport and alkaline phosphatase activity in the chick. Am. J. Physiol., 240, E384-E390.

BITAR K., REINHOLD J. G., 1972. Phytase and alkaline phosphatase activities in the intestinal mucosae of rat, chicken, calf and man. Biochim. biophys. Acta, 268, 442-452.

BOUDAILLIEZ B., 1984. Les métabolites de la vitamine D et les rachitismes, 71-98. In FOURNIER A. et al., Vitamine $D$ et maladies des os et du métabolisme minéral. Masson, Paris. 
CHAN S. D. H., ATKINS D., 1983. Distribution and properties of $\mathrm{Ca}^{2+}$-ATPase, phytase and alkaline phosphatase in isolated enterocytes from normal and vitamin D- deficient rats. Gut, 24, 886892.

DAVIES M. I., RITCEY G. M., MOTZOK I., 1970. Intestinal phytase and alkaline phosphatase of chicks : influence of dietary calcium, inorganic and phytate phosphorus and vitamin $D_{3}$. Poultry Sci., 49, 1280-1286.

DAVIES N. T., FLETT A. A., 1978. The similary between alkaline phosphatase (E.C.3.1.3.1.) and phytase (E.C.3.1.3.8.) activities in rat intestine and their importance in phytate-induced zinc deficiency. Br. J. Nutr., 39, 307-316.

De LUCA H. F., TANAKA Y., BAXTER L. A., 1975. The interrelationship between vitamin D metabolites and phosphate metabolism, 223-237. In AVIOLI L., BORDIER Ph., FLEISH H., MASSRY S., SLATOPOLSKY E., Phosphate metabolism kidney and bone. Pub. Armour Montagu.

GARABEDIAN M., 1984. Les méthodes d'étude du métabolisme de la vitamine $D$ chez l'homme, 41-59. In FOURNIER A., GARABEDIAN M., SEBERT J. L., MEUNIER P. J., Vitamine $D$ et les maladies des os et du métabolisme minéral. Masson, Paris.

GUÉGUEN L., PEREZ J. M., 1981. - A re-evaluation of recommended dietary allowances of calcium and phosphorus for pigs. Proc. Nutr. Soc., 40, 273-278.

HAUSSLER M. R., NAGODE L. A., RASMUSSEN H., 1970. Induction of intestinal brush border alkaline phosphatase activity by vitamin D and identity with Ca-ATPase. Nature, 228, 1199 1201.

KEMPSON S., KIM J., NORTHRUP T., KNOX F., DOUSA T., 1979. Alkaline phosphatase in adaptation to low dietary phosphate intake. Am. J. Physiol., 237, E465-E473.

KRAWITT E. L., KORSON R., 1972. Effect of vitamin D on brush border alkaline phosphatase in the rat small intestine. Enzyme, 13, 278-285.

LOWRY O. H., ROSEBROUGH N. J., FARR A. L., RANDALL R. J., 1951. Protein measurement with the Folin phenol reagent. $J$. biol. Chem., 193, 265-275.

McCARTHY J. T., BARHAM S. S., KUMAR R., 1984. 1,25-dihydroxyvitamin $D_{3}$ rapidly alters the morphology of the duodenal mucosa of rachitic chicks : evidence for novel effects of 1,25 dihydroxyvitamin $D_{3}$. J. Steroid. Biochem., 21, 253-258.

McCUAIG L. W., MOTZOK I., 1972. Regulation of intestinal alkaline phosphatase by dietary phosphate. Can. J. Physiol. Pharmacol., 50, 1152-1156.

MATSUMOTO T., FONTAINE O., RASMUSSEN H., 1980. Effect of 1,25-dihydroxyvitamin $D_{3}$ on phosphate uptake into chick intestinal brush border membrane vesicles. Bioch. biophys. Acta, 599, 13-23.

MOORE R. J., VEUM T. L., 1982. Effect of dietary phosphate and yeast culture level on the utilization of phytate phosphorus by the rat. Nutr. Rep. int., 25, 221-233.

MOORE R. J., VEUM T. L., 1983a. Effect of source and level of dietary yeast product on phytate phosphorus utilization by rats fed low phorphorus diets. Nutr. Rep. int., 27, 1267-1275.

MOORE R. J., VEUM T. L., 1983b. Adaptative increase in phytate digestibility by phosphorusdeprived rats and the relationship of intestinal phytase (E.C.3.1.3.8.) and alkaline phosphatase (E.C.3.1.3.1.) to phytate utilization. Br. J. Nutr., 49, 145-152.

MOORE R. J., REEVES P. G., VEUM T. L., 1984. Influence of dietary phosphorus and sulfaguanidine levels on $\mathrm{P}$ utilization in rats. Br. J. Nutr., 51, 453-465.

MOSER R. L., PEO E. R. Jr., MOSER B. D., LEWIS A. J., 1982. Effect of grain source and dietary level of oat hulls on phosphorus and calcium utilization in the growing pig. J. anim. Sci, 54 , 800-805.

OBERLEAS D., 1971. The determination of phytate and inositol phosphates. Methods biochem. Anal., 20, 87-101.

PETERLIK M., WASSERMAN R., 1980. Regulation by vitamin D of intestinal phosphate absorption. Horm. Metab. Res., 12, 216-219.

PILEGGI V. J., De LUCA H. F., STEENBOCK H., 1955. The role of vitamin D and intestinal phytase in the prevention of rickets in rats on cereal diets. Arch. Biochem. Biophys., 58, 194-204.

POINTILLART A., FONTAINE N., THOMASSET M., 1984a. Hormonal regulation of calcium and phosphorus metabolism in growing pigs fed phytate-rich diets. Calcif. Tissue Int., 36 (suppl. 2), 548. 
POINTILLART A., FONTAINE N., THOMASSET M., 1984b. Phytate phosphorus utilization and intestinal phosphatases in pigs fed low phosphorus: wheat and corn diets. Nutr. Rep. int., 29, 473-483.

POINTILLART A., JAY M. E., FONTAINE N., 1985. Utilisation digestive du phosphore phytique chez le porc en croissance. $J$. Rech. porcine en France, 17, 463-472. Ed. ITP-MNE, Paris.

PREECE M. A., O'RIORDAN J. L. H., LAWSON D. E. M., KODICEK E., 1974. A competitive protein binding assay for 25-hydroxycholecalciferol and 25-hydroxy-ergocalciferol in serum. Clin. chim. Acta, 54, 235-242.

ROBERTS A. H., YUDKINS J., 1961. Effect of phytate and other dietary factors on intestinal phytase and bone calcification in the rat. Brit. J. Nutr., 15, 457-471.

SHIRAZI S., BEECHEY R. B., BUTTERWORTH P. J., 1981. The use of potent inhibitors of alkaline phosphatase to investigate the role of the enzyme in intestinal transport of inorganic phosphate. Biochem. J., 194, 803-809.

STEENBOCK H., KRIEGER C. H., WIEST W. G., PILEGGI V. J., 1953. Vitamin D and intestinal phytase. J. biol. Chem., 205, 993-999.

SZYMANOWICZ A., RANDOUX A., BOREL J. P., 1979. Dosage de l'hydroxyproline totale de l'urine. Ann. Biol. clin., 37, 113-116.

VALINIETSE M. Y., BAUMAN V.K., 1984. Activity of alkaline phosphatase in small intestine mucosa and excrements of chickens as related to vitamin D provision (en russe). S. Kh. Biol., 10, 116120. 